\title{
On the spreading of characteristics for non-convex conservation laws
}

\author{
Helge Kristian Jenssen \\ SISSA, Via Beirut 2-4, 34014 Trieste, Italy (jenssen@sissa.it) \\ Carlo Sinestrari \\ Università di Roma 'Tor Vergata', Via della Ricerca Scientifica, \\ 00133 Roma, Italy (sinestra@mat.uniroma2.it)
}

(MS received 14 July 2000; accepted 04 October 2000)

\begin{abstract}
We study the spreading of characteristics for a class of one-dimensional scalar conservation laws for which the flux function has one point of inflection. It is well known that in the convex case the characteristic speed satisfies a one-sided Lipschitz estimate. Using Dafermos' theory of generalized characteristics, we show that the characteristic speed in the non-convex case satisfies an Hölder estimate. In addition, we give a one-sided Lipschitz estimate with an error term given by the decrease of the total variation of the solution.
\end{abstract}

\section{Introduction}

We consider scalar conservation laws of the form

$$
\partial_{t} u(x, t)+\partial_{x} f(u(x, t))=0, \quad u(x, 0)=u_{0}(x), \quad(x, t) \in \mathbb{R} \times \mathbb{R}^{+} .
$$

The flux function $f$ is assumed to be of class $C^{2}$ and the initial value $u_{0}$ is taken in $B V(\mathbb{R})$. It is well known that this problem is well-posed in the class of the socalled entropy solutions, which are distributional weak solutions satisfying a suitable admissibility condition. While the theory of existence and uniqueness is more or less complete for a general $f$, the qualitative properties of the entropy solutions are not correspondingly well understood, except for the case when $f$ is convex.

In this paper we investigate a possible generalization to the non-convex case of the classical one-sided Lipschitz estimate due to Oleinik [12], which holds in the convex case. Assuming that $f^{\prime \prime}(u) \geqslant c>0$ for all $u$, with $|u| \leqslant \sup _{x \in \mathbb{R}}\left|u_{0}(x)\right|$, the Oleinik estimate says that

$$
u(y, t)-u(x, t) \leqslant \frac{y-x}{c t}, \quad x \leqslant y, \quad t>0 .
$$

We recall that in the case of Burgers' equation (i.e. when $f(u)=\frac{1}{2} u^{2}$ ) this estimate was obtained by Hopf [10]. Estimate (1.2) is interesting for several reasons. In [12], it was used to obtain a uniqueness theorem for entropy solutions based on Holmgren's method. It has provided a powerful tool to study the large-time decay of solutions (see, for example, [8]). It has been applied by Tadmor [14] to obtain local error estimates for approximation schemes. Finally, generalizations of the Oleinik estimate 
to conservation laws with a source term [13] or to genuinely nonlinear systems [2] have been used to study some stability properties of the entropy solutions.

A more accurate analysis (see, for example, $[5,9]$ ) shows that the Oleinik estimate can be regarded as a consequence of the following sharper one, valid when $f^{\prime \prime}(u)$ is non-negative and not identically zero on any interval:

$$
f^{\prime}(u(y, t))-f^{\prime}(u(x, t)) \leqslant \frac{y-x}{t}, \quad x \leqslant y, \quad t>0 .
$$

This form of the inequality has a natural geometric interpretation, since $f^{\prime}(u)$ is the characteristic speed associated with the solution. The inequality bounds the growth of the characteristic speed and thus states that the characteristics cannot spread out arbitrarily fast.

A natural question is whether the form (1.3) of the Oleinik inequality can also hold in the non-convex case. Additionally, one could study the validity of the analogue of (1.3) for multidimensional scalar conservation laws. A negative answer to both issues has been given by Hoff [9], who has shown that, apart from the convex one-dimensional case, estimate (1.3) can fail, and in addition it does not ensure uniqueness (i.e. it is also satisfied by certain weak solutions that are not entropic).

To show that (1.3) does not hold in general, Hoff simply observed that if $f$ has at least two inflection points, then $u$ can have centred rarefaction waves for positive times, and this behaviour is incompatible with the Oleinik estimate. He left open the question of whether (1.3) holds when $f$ has a single inflection point. As we show here, however, the Oleinik estimate is also violated in this case. In fact, when $f$ is non-convex, the solution exhibits, in general, contact discontinuities, and one finds that the ratio $\left[f^{\prime}(u(y, t))-f^{\prime}(u(x, t))\right] /[y-x]$ tends to infinity as $x, y$ approach a contact discontinuity.

The aim of this paper is to provide suitable weakened forms of (1.3) that are also valid in the non-convex case. Although we conjecture that our results can be extended to more general choices of $f$, our analysis here is restricted to the case where $f$ has one inflection point. For definiteness, we assume the following normalization:

$$
f(0)=f^{\prime}(0)=f^{\prime \prime}(0)=0, \quad u f^{\prime \prime}(u)<0 \quad \text { for } u \neq 0 .
$$

A class of model equations is given by the conservation laws

$$
\partial_{t} u(x, t)-\partial_{x}\left[|u(x, t)|^{m} u(x, t)\right]=0,
$$

where $m>1$. The assumption that $f$ has a single inflection point guarantees that no centred rarefaction waves are generated for $t>0$. However, the solution contains, in general, left contact discontinuities along which characteristics are emitted. The fine regularity properties of solutions have been analysed in detail by Dafermos [6], using the theory of generalized characteristics. Even in the case of one inflection point, such an analysis is considerably harder than in the convex case, since the presence of contact discontinuities makes the structure of the solutions more intricate. Nevertheless, Dafermos proved that for a generic class of initial data, the solution is piecewise smooth and therefore has a relatively simple structure. We recall the results of $[6]$ in $\S 2$, since they provide the starting point of our analysis. 
In $\S 3$ we give a first extension of the Oleinik estimate consisting of a one-sided Hölder estimate for $f^{\prime}(u(x, t))$. More precisely, we consider a piecewise smooth solution of (1.1). We show that $f^{\prime}(u(x, t))$ still satisfies a one-sided Lipschitz estimate away from contact discontinuities, while near such a discontinuity it satisfies, for all but finitely many values of $t$, an estimate like (1.3), with the right-hand side replaced by $C \sqrt{y-x}$ for some constant $C$.

In $\S 4$ we give another generalization by adding a suitable remainder term to the right-hand side in (1.3). Namely, we prove that the solution to (1.1) satisfies

$$
f^{\prime}(u(y, t))-f^{\prime}(u(x, t)) \leqslant \frac{y-x}{t}+C\left[T V u_{0}-T V u(\cdot, t)\right], \quad x \leqslant y, \quad t>0,
$$

for some constant $C$. Here, $T V$ denotes the total variation; we recall that the total variation of $u(\cdot, t)$ is non-increasing in time. This result is first proved for piecewise smooth solutions but, unlike the result of $\S 3$, can be extended by density to general $B V$ solutions. Estimate (1.6) shows that, intuitively speaking, when the Oleinik estimate is violated, there is at the same time some cancellation effect that decreases the variation of the solution. Since the total variation of the solution necessarily tends to some non-negative constant as $t \rightarrow \infty$, we can easily deduce that $u$ satisfies an 'asymptotic' Oleinik estimate (see corollary 4.4). Let us observe that an estimate similar to (1.6) was proved by Bressan and Colombo [1] for genuinely nonlinear systems of conservation laws; in their estimate the total variation is replaced by the Glimm interaction functional.

Let us finally mention that estimates of a different type on the total variation of $f^{\prime}(u(x, t))$ or of other nonlinear functions of $u$ have been obtained by Dafermos [6], Cheng [3], Zumbrun [16], Tegnander [15] and Cheverry [4].

\section{Preliminaries}

We define an entropy $B V$ solution of (1.1) to be a bounded measurable function defined on $\mathbb{R} \times \mathbb{R}^{+}$, with distributional derivatives $\partial_{t} u, \partial_{x} u$, which are locally finite Borel measures, satisfying the entropy inequality

$$
\partial_{t} \eta(u(x, t))+\partial_{x} q(u(x, t)) \leqslant 0,
$$

for every pair $\eta, q \in C^{2}(\mathbb{R})$ such that $\eta$ is convex and $q^{\prime}=\eta^{\prime} f^{\prime}$. For initial data $u_{0}$ in $L^{\infty} \cap B V_{\text {loc }}$, it is well known that (1.1) has a unique entropy $B V$ solution (see [11]). Moreover, if $u_{0}$ has finite total variation, then the total variation of $u(\cdot, t)$ is also finite and is non-increasing in $t$. We normalize the solution to be continuous from the left.

The structure of the solutions to (1.1) under assumptions (1.4) has been studied by Dafermos [6], whose results will be the starting point for our analysis. In the remainder of this section, we recall some definitions and theorems from that paper and prove some more results needed for our later analysis.

The key ingredient of the analysis of [6] is the notion of generalized characteristic. We recall that a (generalized) characteristic associated with an entropy $B V$ solution $u(x, t)$ of $(1.1)$ is a Lipschitz curve $\xi:\left[t_{0}, t_{1}\right] \rightarrow \mathbb{R}, 0 \leqslant t_{0}<t_{1}<\infty$, which satisfies the differential inclusion

$$
\dot{\xi}(t) \in\left[f^{\prime}(u(\xi(t)+, t)), f^{\prime}(u(\xi(t), t))\right]
$$


for almost all $t \in\left[t_{0}, t_{1}\right]$. From the theory of differential equations with discontinuous right-hand side [7], it follows that, given any point $(\bar{x}, \bar{t}) \in \mathbb{R} \times(0, \infty)$, there is a funnel of generalized characteristics passing through $(\bar{x}, \bar{t})$, lying between a minimal and a maximal one.

Examples of characteristics are classical characteristics and shock curves. We recall that a characteristic $\xi$ is called classical if there exists $\bar{u} \in \mathbb{R}$ such that, for all $t \in\left[t_{0}, t_{1}\right]$,

$$
\xi(t)=\xi\left(t_{0}\right)+f^{\prime}(\bar{u})\left(t-t_{0}\right), \quad u(\xi(t), t)=u(\xi(t)+, t)=\bar{u} .
$$

A characteristic $\chi$ is called a shock if $u(\chi(t), t) \neq u(\chi(t)+, t)$ for a.e. $t \in\left[t_{0}, t_{1}\right]$. It is well known that the speed of a shock satisfies a.e. the so-called Rankine-Hugoniot relation

$$
\dot{\chi}(t)=\frac{f^{\prime}(u(\chi(t), t))-f^{\prime}(u(\chi(t)+, t))}{u(\chi(t), t)-u(\chi(t)+, t)} .
$$

In addition, the values of the solution on the two sides of the shock must satisfy the Oleinik (E)-condition. Setting $u_{-}=u(\chi(t), t)$ and $u_{+}=u(\chi(t)+, t)$, this condition says that the segment joining the points $\left(u_{-}, f\left(u_{-}\right)\right)$and $\left(u_{+}, f\left(u_{+}\right)\right)$of the graph of $f$ must lie above (respectively, below) the graph of $f$ if $u_{-}>u_{+}$(respectively, $u_{+}>u_{-}$). To see how the Rankine-Hugoniot and the Oleinik (E)-condition can be derived from the entropy inequality (2.1), see, for example, [6, lemmas 2.2, 2.9]. A consequence of the Oleinik (E)-condition is that, along a shock curve $\chi$,

$$
f^{\prime}(u(\chi(t)+, t)) \leqslant \dot{\chi}(t) \leqslant f^{\prime}(u(\chi(t), t) .
$$

By a genuine shock we mean a curve of discontinuity $x=\chi(t), t \in[a, b]$, for which the two inequalities above are strict for almost all $t \in[a, b]$. Recalling (2.3), one sees that, for a strictly convex flux function $f$, the two inequalities are necessarily strict, while for a general flux, one or both of them can be equalities. For the fluxes we consider in this paper, which have one inflection point and satisfy the normalization (1.4), it is easy to see that the first inequality in (2.4) is necessarily strict, while the second one can be an equality. If a curve of discontinuity $x=\chi(t)$, $t \in[a, b]$, satisfies $\dot{\chi}(t)=f^{\prime}(u(\chi(t), t))$ for almost all $t \in[a, b]$, we call it a left contact discontinuity. From the definition, it follows that on both sides of a genuine shock the characteristics go inside the shock. On the other hand, characteristics can be emanated tangentially from the left side of a contact discontinuity, and this feature makes the structure of the solution in the non-convex case more difficult to study. However, for a generic set of initial data, the solution is piecewise smooth and thus has a simpler structure. Here, the property 'generic' is meant with respect to the standard $C^{\infty}$ topology.

Theorem 2.1 (cf. theorem 3.1 of [6]). Assume that $f, u_{0}$ are $C^{\infty}$ smooth and that assumptions (1.4) are satisfied. Then, for a generic set of initial data, the domain $\mathbb{R} \times[0, \infty)$ of the entropy solution of (1.1) consists of four disjoint subsets $\mathcal{D}, \mathcal{S}$, $\mathcal{W}, \mathcal{I}$, with the following properties.

(a) $\mathcal{D}$ is a dense set on which $u(x, t)$ is $C^{\infty}$.

(b) $\mathcal{S}$ is a finite union of $C^{\infty}$ arcs that are genuine shocks or left contact discontinuities. 
(c) $\mathcal{W}$ is a finite union of characteristics that are weak waves, i.e. lines along which the solution is continuous but one of its derivatives has a jump discontinuity.

(d) $\mathcal{I}$ is the finite set of shock generation points, shock interaction points and points of interaction between shocks and weak waves.

The extremal backward characteristics have some special properties, which are described in [6]. We state here these properties in the case of a piecewise smooth solution, which is enough for our purposes.

TheOREM 2.2 (cf. theorems 2.1, 2.2 of [6]). Let $u$ be a piecewise smooth solution of (1.1), as in theorem 2.1. Given $(\bar{x}, \bar{t}) \in \mathbb{R} \times(0, \infty)$, let $\xi$ and $\zeta$ be the maximal and minimal backward characteristic through $(\bar{x}, \bar{t})$. If $u(\bar{x}, \bar{t})=u(\bar{x}+, \bar{t})=0$, then $\xi$ and $\zeta$ are classical and satisfy $\xi(t) \equiv \zeta(t) \equiv \bar{x}$. If $u(\bar{x}, \bar{t}) \neq 0$ or $u(\bar{x}+, \bar{t}) \neq 0$, then the following properties hold.

(i) There is a finite mesh $0=t_{0}<t_{1}<\cdots<t_{n+1}=\bar{t}$ such that $\xi$ is a convex polygonal with vertices at the points $\left(\xi\left(t_{j}\right), t_{j}\right), j=0, \ldots, n+1$. Furthermore, $\xi$ is classical in every interval $\left(t_{j-1}, t_{j}\right)$ and crosses a contact discontinuity at each vertex $\left(\xi\left(t_{j}\right), t_{j}\right)$.

(ii) $u(\zeta(t), t)$ is a continuous function with constant sign on $[0, \bar{t}]$, which is nondecreasing when $u(\bar{x}, \bar{t})<0$ and non-increasing when $u(\bar{x}, \bar{t})>0$. For each $t \in(0, \bar{t})$,

$$
\dot{\zeta}(t)=f^{\prime}(u(\zeta(t), t)),
$$

so that $\zeta$ is a convex $C^{1}$ curve. In addition, there is a finite mesh $0=s_{0}<$ $s_{1}<\cdots<s_{m+1}=\bar{t}$ such that, on the intervals $\left(s_{i-1}, s_{i}\right)$, the curve $\zeta$ is alternately a classical characteristic and a contact discontinuity.

The following result for maximal forward characteristics shows that once a discontinuity has developed, it propagates for all later times.

TheOREm 2.3 (cf. theorem 2.3 of $[6]$ ). Let $(\bar{x}, \bar{t}) \in \mathbb{R} \times(0, \infty)$ be a point of discontinuity of an entropy solution $u$ and let $\chi$ denote the maximal forward characteristic starting from $(\bar{x}, \bar{t})$. Then $\chi$ is a shock on $[\bar{t}, \infty)$. Also, the functions $u(\chi(t), t)$, $u(\chi(t)+, t)$ are continuous from the right on $[\bar{t}, \infty)$.

One of the assumptions required on the generic set of initial data of theorem 2.1 is that $u_{0}$ changes sign across a finite number of points $-\infty<y_{1}<\cdots<y_{l}<\infty$. We assume that this property holds and denote by $\phi_{i}$ the maximal forward characteristic starting at $\left(y_{i}, 0\right)$. If $\phi_{i-1}$ and $\phi_{i}$ collide, we let $s_{i}^{*}>0$ denote the time of collision. In that case, we have $\phi_{i-1}(t)=\phi_{i}(t)$ for all $t \in\left[s_{i}^{*}, \infty\right)$. The characteristics $\phi_{i}$ partition the upper half-plane in $l+1$ regions $\mathcal{R}_{1}, \ldots, \mathcal{R}_{l+1}$ given by

$$
\mathcal{R}_{i}=\left\{(x, t) \mid 0 \leqslant t<s_{i}^{*}, \phi_{i-1}(t)<x<\phi_{i}(t)\right\}, \quad i=1, \ldots, l+1,
$$

where we let $\phi_{0} \equiv-\infty, \phi_{l+1} \equiv \infty$ and $s_{i}^{*}=\infty$ whenever $\phi_{i-1}(t)<\phi_{i}(t)$ for all $t \in[0, \infty)$. It follows that any minimal backward characteristic from a point in $\mathcal{R}_{i}$ remains within $\mathcal{R}_{i}$ and so, by theorem 2.2 (ii), the sign of $u(x, t)$ is constant on $\mathcal{R}_{i}$. 
Each curve $\phi_{i}$ starts out at time $t=0$ as a classical characteristic with zero speed. It can either remain constant for all times or it can collide with a shock at some later time $t=t_{i}^{*}$; then it remains a shock for all $t \in\left(t_{i}^{*}, s_{i}^{*}\right)$, by theorem 2.3. Generically, the interval $\left(t_{i}^{*}, s_{i}^{*}\right)$ can be partitioned in a finite number of non-degenerate subintervals, where $\phi_{i}$ is alternately a genuine shock and a left contact discontinuity. Since the solution changes sign across a left contact discontinuity, these parts of the curves $\phi_{i}$ are the only left contacts present in the solution.

It is convenient to further divide each region $\mathcal{R}_{i}$ into two subregions. The idea is to identify where the solution behaves as in the case of a convex flux and where the behaviour is influenced by the presence of the inflection point of $f$. For any $t<s_{i}^{*}$, we let $\sigma_{i}(t)$ be the point of $\left[\phi_{i-1}(t), \phi_{i}(t)\right]$ such that the maximal backward characteristic through a point $(x, t)$ lies strictly to the left of $\phi_{i}$ on $[0, t]$ if $x \in\left[\phi_{i-1}(t), \sigma_{i}(t)\right)$, whereas it meets $\phi_{i}$ at some positive time if $x \in\left[\sigma_{i}(t), \phi_{i}(t)\right)$. We now define the following subregions,

$$
\begin{aligned}
\mathcal{G}_{i} & =\left\{(x, t) \in \mathbb{R} \times \mathbb{R}^{+} \mid \phi_{i-1}(t)<x<\sigma_{i}(t)\right\}, \\
\mathcal{H}_{i} & =\left\{(x, t) \in \mathbb{R} \times \mathbb{R}^{+} \mid \sigma_{i}(t)<x<\phi_{i}(t)\right\},
\end{aligned}
$$

where $\phi_{l+1} \equiv \infty$. We refer to $\mathcal{G}_{i}, \mathcal{H}_{i}, i=1, \ldots, l+1$, as the convex and non-convex regions of the solution, respectively. This terminology is not related to the shape of the regions, but to the behaviour of the solution. In fact, the domain of dependence of $\mathcal{G}_{i}$ is the interval $\left(y_{i-1}, y_{i}\right)$, where $u_{0}$ does not cross the inflection point of $f(u)$. It follows that the behaviour of $u(x, t)$ on $\mathcal{G}_{i}$ is governed by the same mechanism as in the case of convex equations. We thus have that the Oleinik estimate (1.3) holds for points in a convex region.

Proposition 2.4. For $(x, t),(y, t) \in \mathcal{G}_{i}$ with $x \leqslant y$, the solution $u(x, t)$ satisfies the one-sided Lipschitz estimate

$$
f^{\prime}(u(y, t))-f^{\prime}(u(x, t)) \leqslant \frac{y-x}{t} .
$$

Proof. We consider the case when $u(\cdot, t)$ is continuous at $x$ and $y$, since the general case follows by approximation. Let us denote by $\zeta_{1}$ and $\zeta_{2}$ the maximal backward characteristics from $(x, t)$ and $(y, t)$, respectively. These characteristics cannot cross any contact discontinuity, by the definition of $\mathcal{G}_{i}$, and so in both cases the mesh of theorem 2.2(i) is the trivial one $t_{0}=0, t_{1}=t$. Thus we have $\zeta_{1}(s)=x-f^{\prime}(u(x, t))(t-s), \zeta_{2}(s)=y-f^{\prime}(u(y, t))(t-s)$. Since $\zeta_{1}$ and $\zeta_{2}$ cannot cross, we have $\zeta_{1}(0) \leqslant \zeta_{2}(0)$, that is,

$$
t\left[f^{\prime}(u(y, t))-f^{\prime}(u(x, t))\right] \leqslant y-x .
$$

We cannot use the same argument for the non-convex regions $\mathcal{H}_{i}$, since these regions are spanned by characteristics emanating from contact discontinuities. To investigate the validity of Oleinik-type estimates in these regions, we must therefore analyse how the spreading of characteristics is affected by the presence of contact discontinuities. We collect in the rest of this section some preliminary properties of the curves $\phi_{i}$, which will be used in the analysis of the next sections. 
Let us introduce some more notation and terminology. We denote by $J_{i}$ the set of times $t \in\left(t_{i}^{*}, s_{i}^{*}\right)$ at which $\phi_{i}$ is a left contact discontinuity, i.e. such that

$$
f^{\prime}\left(u\left(\phi_{i}(t), t\right)\right)=\frac{f\left(u\left(\phi_{i}(t)+, t\right)\right)-f\left(u\left(\phi_{i}(t), t\right)\right)}{u\left(\phi_{i}(t)+, t\right)-u\left(\phi_{i}(t), t\right)} .
$$

The interior of $J_{i}$ is denoted by $J_{i}^{\text {o }}$. It was proved in [6] that $\phi_{i}$ is generically of class $C^{\infty}$ on $J_{i}^{\text {o }}$, except at a finite number of points across which some derivative $\phi_{i}^{(m)}, m \geqslant 1$, experiences a jump discontinuity. Moreover, we say that a classical characteristic is grazing upon $\phi_{i}$ at time $t=\tau$ if it is defined in an interval $\left[t_{0}, t_{1}\right]$ with $t_{0}<\tau<t_{1}$ and is tangent to $\phi_{i}$ at $\left(\phi_{i}(\tau), \tau\right)$.

Proposition 2.5. Let $t_{0}, t_{1}$ be such that $\left[t_{0}, t_{1}\right] \subset J_{i}^{\mathrm{o}}$. Then, generically, the following properties hold.

(i) Setting $\Gamma=\left\{(x, t): t \in\left[t_{0}, t_{1}\right], x=\phi_{i}(t)\right\}$, there exists a neighbourhood $N$ of $\Gamma$ such that $u$ is continuous at the points of $N$ not lying on the curve $\phi_{i}$.

(ii) There exists $\varepsilon>0$ with the following property: for all $t \in\left[t_{0}, t_{1}\right]$, the line $\xi(s)=\phi_{i}(t)+(s-t) \dot{\phi}_{i}(t)$ is a classical characteristic at least in the interval $s \in[t, t+\varepsilon]$.

(iii) Setting $u_{ \pm}(t):=u\left(\phi_{i}(t) \pm, t\right)$ for $t \in\left[t_{0}, t_{1}\right]$, both $u_{-}(t)$ and $u_{+}(t)$ have constant sign, one opposite to the other, and both $\left|u_{-}(t)\right|$ and $\left|u_{+}(t)\right|$ are strictly decreasing.

(iv) Call $\tau_{0}$ the infimum of the times $t$ such that $\left[t, t_{1}\right] \subset J_{i}$. Then $\left[\tau_{0}, t_{1}\right] \subset J_{i}$ and there is a grazing ray upon $\phi_{i}$ at time $\tau_{0}$.

Proof. We recall that, by [6, corollary 2.2], no shock can collide with $\phi_{i}$ at any $t \in J_{i}$. The number of shocks is generically finite, and so we can find a neighbourhood $N$ of the set $\Gamma$ where the solution has no other points of discontinuity except those on $\phi_{i}$. This proves (i).

Let us now set $N^{\prime}=\left\{(x, t) \in N: x<\phi_{i}(t)\right\}$. Since $u$ is continuous in $N^{\prime}$, such a set is covered univalently by classical characteristics. Characteristics can, in principle, either be absorbed or be emanated by $\phi_{i}$; however, by [6, lemma 3.6], there is generically only a finite number of characteristics that are absorbed by $\phi_{i}$. But then there are no absorbed characteristics at all, since otherwise they would cross the emanated ones and $N^{\prime}$ would not be covered univalently. Thus we can find $\varepsilon$ as in (ii).

The functions $u_{-}(t)$ and $u_{+}(t)$ have constant sign because $u$ has constant sign in each region $\mathcal{R}_{i}$. In addition, $f^{\prime}\left(u_{-}(t)\right)=\phi_{i}^{\prime}(t)$ is strictly increasing in $\left[t_{0}, t_{1}\right]$ by [6, lemma 3.4]. Recalling that $f$ satisfies (1.4) and that $u_{-}(t)$ and $u_{+}(t)$ are related by (2.8), we deduce that $\left|u_{-}(t)\right|$ and $\left|u_{+}(t)\right|$ are strictly decreasing.

It remains to prove (iv). We have that $\left.] \tau_{0}, t_{1}\right] \subset J_{i}$ by definition of $\tau_{0}$. Since $u_{+}$ and $u_{-}$are continuous from the right, $\tau_{0}$ also belongs to $J_{i}$. By $\left[6\right.$, corollary 2.2], $\phi_{i}$ is differentiable at $\tau_{0}$ and $\dot{\phi}_{i}\left(\tau_{0}\right)=f^{\prime}\left(u_{-}\left(\tau_{0}\right)\right)$. Let us set, for simplicity, $x_{0}=\phi_{i}\left(\tau_{0}\right)$. We have to show that the line $\xi_{0}(s):=x_{0}+\left(s-\tau_{0}\right) \dot{\phi}_{i}\left(\tau_{0}\right)$ is a classical characteristic for $s$ in a neighbourhood of $\tau_{0}$. By part (ii), this is certainly true for $s \in\left[\tau_{0}, \tau_{0}+\varepsilon\right]$. To take into account times smaller than $t$, let us consider the minimal backward 


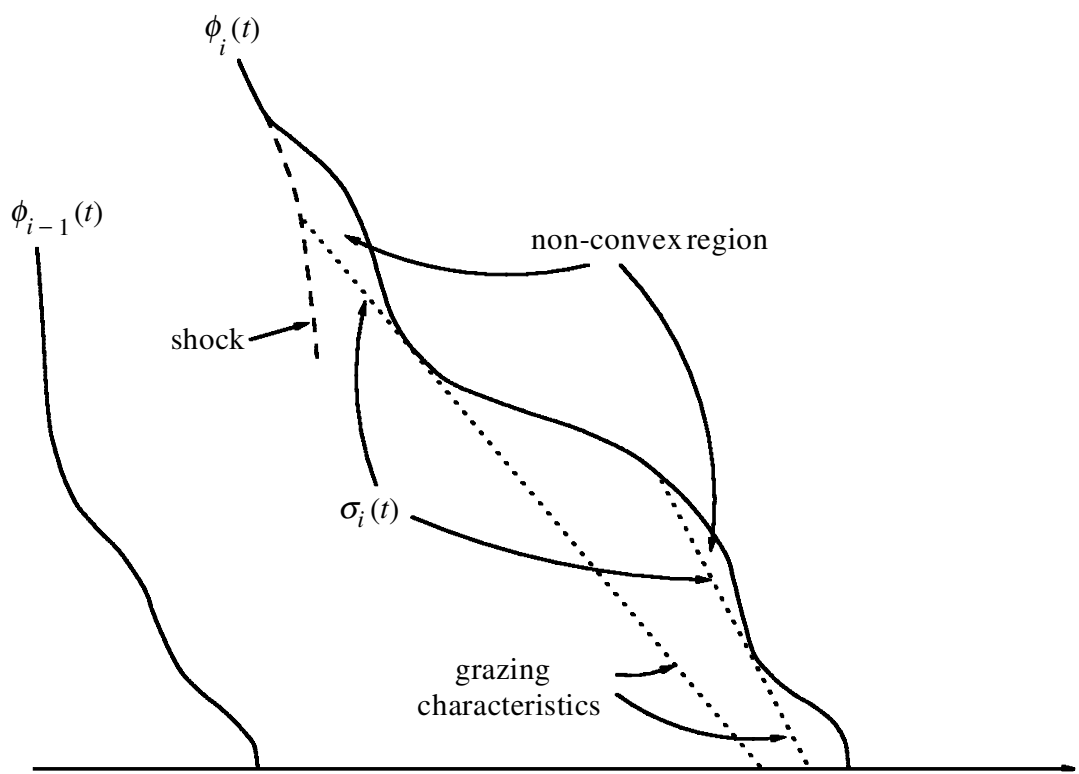

Figure 1. The characteristics bounding non-convex regions.

characteristic from $\left(x_{0}, \tau_{0}\right)$. By theorem $2.2(\mathrm{ii})$, there exists an interval $\left[\tau_{0}-\delta, \tau_{0}\right]$ where such a characteristic is either classical or a contact discontinuity. The latter possibility is excluded, since it would imply that $\left[\tau_{0}-\delta, \tau_{0}\right] \subset J_{i}$, contradicting the definition of $\tau_{0}$. We deduce that the backward characteristic is classical and coincides with $\xi_{0}$, which is therefore a grazing ray upon $\phi_{i}$ at $t_{0}$.

Thus the curve $\phi_{i}$ is alternately a genuine shock, with characteristics impinging upon it from both sides, and a left contact discontinuity, with characteristics emitted to the left. At the left endpoint of a time-interval where $\phi_{i}$ is a left contact, there is a grazing ray upon $\phi_{i}$. Figure 1 shows a typical situation.

\section{Hölder estimate for $f^{\prime}(u)$}

We now start our analysis of the behaviour of the characteristic speed $f^{\prime}(u)$ in the non-convex regions $\mathcal{H}_{i}$. In this section we show that $f^{\prime}(u)$ satisfies no one-sided Lipschitz estimate of the form (1.3), but rather a Hölder estimate with exponent $\frac{1}{2}$. We assume throughout that the solution behaves as in the generic case described by theorem 2.1 .

Let us choose one of the non-convex regions $\mathcal{H}_{i}$ and simplify notation by dropping the subscript $i$. Thus

$$
\sigma(t) \equiv \sigma_{i}(t), \quad \phi(t) \equiv \phi_{i}(t), \quad J \equiv J_{i}, \quad \text { etc. }
$$

We fix a time $t$ for which $\sigma(t)<x<\phi(t)$, and define

$$
\theta(x)=f^{\prime}(u(x, t)), \quad \sigma(t)<x<\phi(t) .
$$


From [6, lemma 3.5], we deduce that the function $\theta$ is increasing and continuous; in addition, it is $C^{\infty}$ smooth, except at the points lying on weak waves, whose number is finite by theorem 2.1 (c).

Let us consider a point $(x, t)$ with $x \in(\sigma(t), \phi(t))$. By the definition of the non-convex regions, the minimal backward characteristic through $(x, t)$ is emitted tangentially from $\phi$ at some previous time, which we denote by $\tau(x)$. The function $\tau$ is increasing in $x$ and satisfies

$$
\begin{aligned}
f^{\prime}(u(x, t)) & =f^{\prime}(u(\phi(\tau(x)), \tau(x)))=\dot{\phi}(\tau(x)), \\
x & =\phi(\tau(x))+\dot{\phi}(\tau(x))(t-\tau(x)) .
\end{aligned}
$$

At the points where $\tau$ is differentiable, we obtain

$$
\ddot{\phi}(\tau(x)) \tau^{\prime}(x)=\frac{1}{t-\tau(x)} .
$$

Recall that $\theta(x)=f^{\prime}(u(x, t))=\dot{\phi}(\tau(x))$, so that

$$
\theta^{\prime}(x)=\frac{1}{t-\tau(x)} .
$$

By continuity, this equality holds at all points of differentiability of $\theta$, and thus yields information about the Lipschitz continuity of $\theta$. We can first observe that, since $\tau(x)$ is strictly positive, the one-sided estimate (1.3) does not hold in the nonconvex regions, at least not with a Lipschitz constant $1 / t$. On the other hand, we see that $\theta$ is Lipschitz continuous if $\tau(x)$ is bounded away from $t$. This is the case if $x$ is bounded away from $\phi(t)$, that is, if we are far from the contact discontinuities, but does not hold in general as we approach such curves. More precisely, let us set

$$
J^{*}:=\{t:(t-\varepsilon, t) \subset J \text { for some } \varepsilon>0\},
$$

where $J=J_{i}$ is the set introduced before proposition 2.5. Using proposition 2.5 (ii), we obtain that

$$
\lim _{x \uparrow \phi(t)} \tau(x)=t \quad \Leftrightarrow \quad t \in J^{*} .
$$

Therefore, if $t \in J^{*}$, no one-sided Lipschitz estimate from above holds for $\theta(x)$ in a left neighbourhood of $(\phi(t), t)$.

We now proceed to estimate the rate at which $\theta^{\prime}(x) \uparrow+\infty$ as $x \uparrow \phi(t)$ with $t \in J^{*}$. We choose $\varepsilon>0$ such that $(t-\varepsilon, t) \subset J$ and that $\phi$ is $C^{\infty}$ smooth in $(t-\varepsilon, t)$; then we take $\eta>0$ such that, for all $x \in(\phi(t)-\eta, \phi(t))$, we have $\tau(x) \in(t-\varepsilon, t)$. Then there are no weak waves crossing the interval $(\phi(t)-\eta, \phi(t))$ at time $t$ and $\theta$ is $C^{\infty}$ smooth in this interval. Differentiating (3.4) once more with respect to $x$ and using (3.3), we obtain

$$
\frac{\theta^{\prime \prime}(x)}{\left(\theta^{\prime}(x)\right)^{3}}=\frac{1}{\ddot{\phi}(\tau(x))} .
$$

Assuming further that there exists $C^{\prime}$ such that $\ddot{\phi}(\tau) \leqslant C^{\prime}$ for $t-\varepsilon<\tau<t$, we obtain the differential inequality

$$
\frac{\theta^{\prime \prime}(x)}{\left(\theta^{\prime}(x)\right)^{3}} \geqslant \frac{1}{C^{\prime}}
$$


for all $x$ sufficiently close to $\phi(t)$. Integrating from $x$ to $\phi(t)$ gives

$$
\frac{1}{\left(\theta^{\prime}(x)\right)^{2}} \geqslant \frac{2}{C^{\prime}}(\phi(t)-x),
$$

where we have used the fact that $\theta^{\prime}(\phi(t))=+\infty$. Solving for $\theta^{\prime}(x)$ and integrating once more gives the estimate

$$
C \sqrt{\phi(t)-x} \geqslant \theta(\phi(t))-\theta(x)=f^{\prime}(u(\phi(t), t))-f^{\prime}(u(x, t))
$$

for $x<\phi(t)$ close to $\phi(t)$ and where $C=\sqrt{2 C^{\prime}}$. Let us summarize the results obtained so far.

Theorem 3.1. Assume that the flux $f \in C^{\infty}$ satisfies conditions (1.4). Assume also that the solution to (1.1) is piecewise smooth, as in the generic case described in theorem 2.1. Given a point $(x, t) \in \mathbb{R} \times \mathbb{R}_{+}$, the following two properties are equivalent.

(i) There exists no neighbourhood $N$ of $x$ such that the ratio $\left[f^{\prime}\left(u\left(x_{2}, t\right)\right)-\right.$ $\left.f^{\prime}\left(u\left(x_{1}, t\right)\right)\right] /\left[x_{2}-x_{1}\right]$ is bounded from above for all $x_{1}, x_{2}$ in $N$ with $x_{2}>x_{1}$.

(ii) There exists a contact discontinuity curve $\phi$ such that $t \in J^{*}$ and $x=\phi(t)$.

In addition, if (ii) holds and if $\limsup _{\tau \uparrow t} \ddot{\phi}_{i}(\tau)<+\infty$, then $f^{\prime}(u(\cdot, t))$ satisfies a Hölder estimate with exponent $\frac{1}{2}$ of the form (3.7) in a left neighbourhood of $x$.

In the previous computations, we have seen that the validity of a Hölder estimate near a point on the curve $\phi$ is related to the curvature of $\phi$ at that point. To better understand the behaviour of the curvature of a left contact discontinuity, we now proceed with a more detailed analysis, which in turn yields a more precise Hölder estimate. For our further analysis we need to assume that the graph of the flux is not too flat at the point of inflection, in the sense that we describe now. From assumptions (1.4), it follows that for each $u \neq 0$ there exists a unique $u^{*} \neq u$ with the property that

$$
f^{\prime}(u)=\frac{f\left(u^{*}\right)-f(u)}{u^{*}-u} .
$$

This is the relation satisfied across a left contact discontinuity with left state $u$ and right state $u^{*}$. For $u \neq 0$, let us define

$$
\rho(u)=\frac{f^{\prime}(u)}{f^{\prime}\left(u^{*}\right)} .
$$

Then $0<\rho(u)<1$ for every $u$; our assumption is that $\rho$ satisfies, in addition,

$$
\operatorname{sgn}(u) \rho^{\prime}(u) \leqslant 0 \quad \text { for } u \neq 0 .
$$

As an example, consider the model equation (1.5). In this case, $\rho$ is constant and equal to the unique positive root of the algebraic equation

$$
m \rho^{1+1 / m}+(m+1) \rho-1=0 .
$$

Under the above assumption, and with the same notation used for proving theorem 3.1, we let $\xi$ and $\zeta$ denote the maximal backward characteristics through $(x, t)$ 


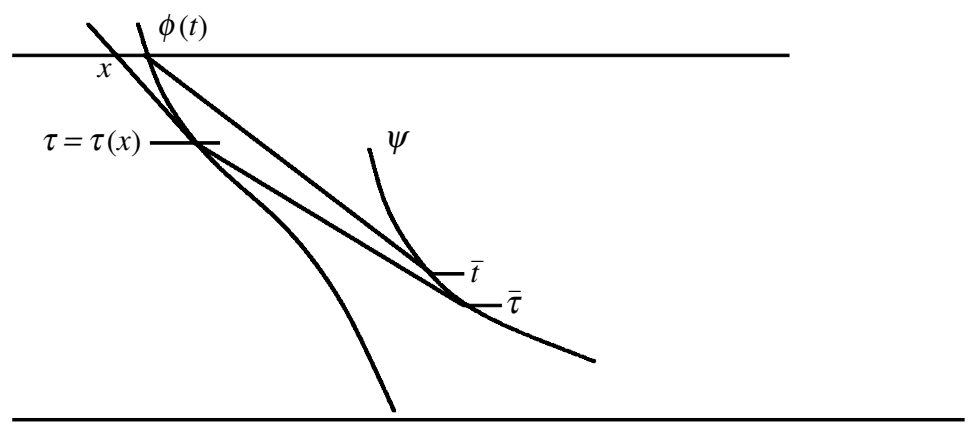

Figure 2. Case A.

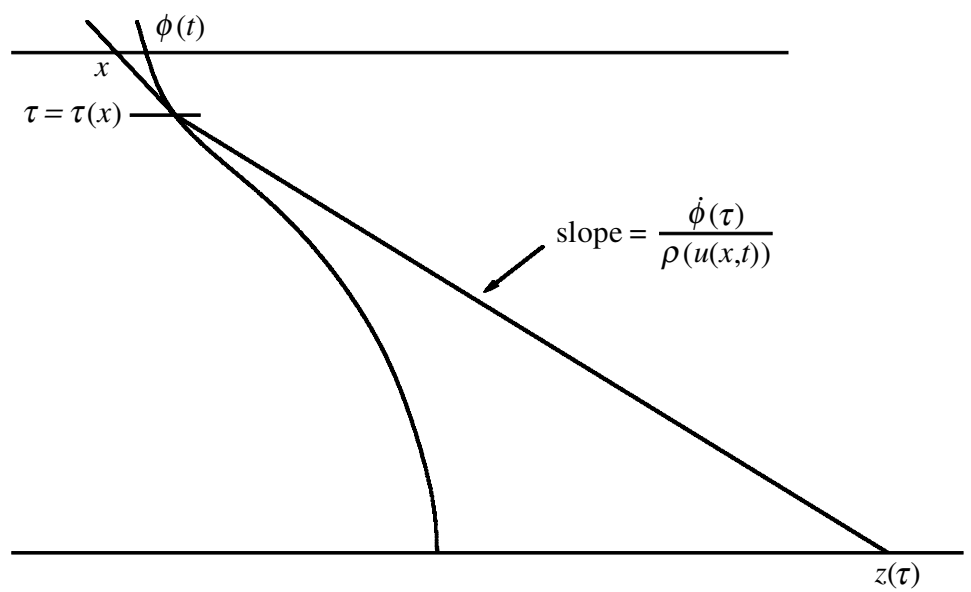

Figure 3. Case B.

and $(\phi(t), t)$, respectively. According to theorem 2.2, these are convex polygonal lines with vertices at the points where they cross left contact discontinuities. By choosing $x<\phi(t)$ sufficiently close to $\phi(t)$, we can assume that one of the following two cases occurs.

(A) Both $\xi$ and $\zeta$ are emitted from the same left contact discontinuity, denoted by $\psi$, at some earlier times $\bar{t}$ and $\bar{\tau}$, respectively. Here, $\bar{t}$ depends on $t$ and $\bar{\tau}$ depends on $\tau=\tau(x)$ (see figure 2).

(B) Both $\xi$ and $\zeta$ are straight line characteristics starting at time zero (see figure 3$)$.

CASE A. Recalling that $u$ is normalized to be continuous from the left, we set

$$
v(\tau)=u(\phi(\tau), \tau)
$$

Referring to figure 2, we have

$$
\phi(\tau)=\dot{\psi}(\bar{\tau})(\tau-\bar{\tau})+\psi(\bar{\tau})=\frac{\dot{\phi}(\tau)}{\rho(v(\tau))}(\tau-\bar{\tau})+\psi(\bar{\tau})
$$


Differentiating with respect to $\tau$ and using (3.10) and the fact that $|v(\tau)|$ is non-increasing, we conclude that

$$
(\rho(v(\tau))-1) \dot{\phi}(\tau) \geqslant \ddot{\phi}(\tau)(\tau-\bar{\tau}) .
$$

Provided that $\phi$ is not intersected by $\psi$ at time $t$, we have $\bar{t}<t$. Letting $\tau \uparrow t$ in (3.12) shows that $\ddot{\phi}$ is uniformly bounded on a backward interval $(t-\varepsilon, t), \varepsilon>0$, so that an estimate of the form (3.7) holds.

On the other hand, if $\rho$ is constant, then (3.12) holds with equality. In this case, if $\phi$ is intersected by $\psi$ at time $t$, then $\tau-\bar{\tau} \rightarrow 0$ and (3.12) implies that $\ddot{\phi}(\tau) \uparrow \infty$ as $\tau \uparrow t$. Thus the curvature of a left contact discontinuity may be infinite, but only at times where it is intersected from the right by another left contact discontinuity. Since all contact discontinuities are contained in the curves $\phi_{i}$, there are only finitely many such intersections.

To proceed, we assume that $t$ is not a time of intersection between $\phi$ and $\psi$. Defining $\rho_{0}=\inf \left\{\rho(v):|v| \leqslant\left\|u_{0}\right\|_{\infty}\right\}>0$ and observing that $\tau(x)-\bar{\tau}>\tau(x)-\bar{t}>0$ for $x$ close enough to $\phi(t)$, we conclude that

$$
\frac{1}{\ddot{\phi}(\tau(x))} \geqslant\left(\frac{1}{\rho_{0}-1}\right) \frac{\tau(x)-\bar{t}}{\dot{\phi}(\tau(x))} .
$$

Substituting this into (3.6), we have the differential inequality

$$
\frac{\mathrm{d}}{\mathrm{d} x}\left(\frac{\theta(x)}{\theta^{\prime}(x)}\right) \geqslant \frac{\rho_{0}}{\rho_{0}-1}-\frac{\theta^{\prime}(x)(t-\bar{t})}{\rho_{0}-1} .
$$

Case B. In this case, we have

$$
\phi(\tau)=\frac{\dot{\phi}(\tau)}{\rho(v(\tau))} \tau+z(\tau),
$$

where $z(\tau)$ is the intersection of the characteristic $\xi$ with the $x$-axis (see figure 3 ). Differentiating with respect to $\tau$, using the properties of $\rho$ and the fact that $z^{\prime}(\tau) \geqslant 0$ gives

$$
\dot{\phi}(\tau) \geqslant \frac{\ddot{\phi}(\tau)}{\rho(v(\tau))} \tau+\frac{\dot{\phi}(\tau)}{\rho(v(\tau))} .
$$

We conclude that

$$
\ddot{\phi}(\tau) \leqslant\left(1-\rho_{0}\right) \frac{|\dot{\phi}(\tau)|}{\tau} .
$$

Substituting this into (3.6), we obtain

$$
\frac{\mathrm{d}}{\mathrm{d} x}\left(\frac{\theta(x)}{\theta^{\prime}(x)}\right) \geqslant \frac{\rho_{0}}{\rho_{0}-1}-\frac{\theta^{\prime}(x) t}{\rho_{0}-1},
$$

which is the same as $(3.13)$ when $\bar{t}=0$. We can thus treat the two cases by the same analysis.

Integrating (3.13) from $x$ to $\phi(t)$ yields the following differential inequality for $\theta(x)$ :

$$
\theta^{\prime}(x) \leqslant \frac{\left(1-\rho_{0}\right) \theta(x)}{\rho_{0}(\phi(t)-x)-(t-\bar{t})(\dot{\phi}(t)-\theta(x))} .
$$




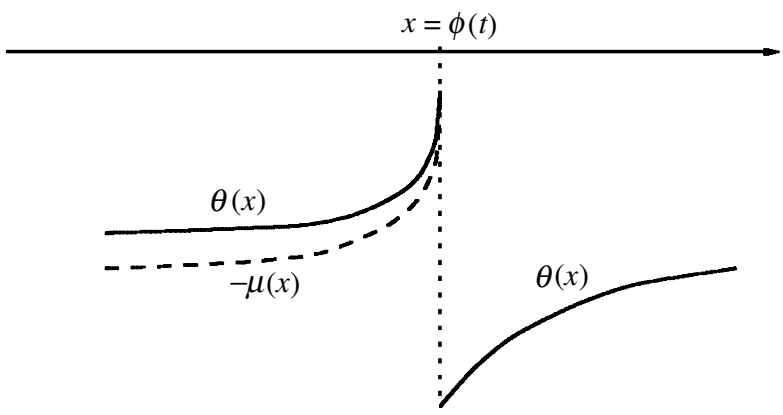

Figure 4. Profile of $\theta(x)$ and $-\mu(x)$.

Letting $\mu(x)$ be the solution of the problem

$$
\mu^{\prime}(x)=\frac{\left(1-\rho_{0}\right) \mu(x)}{\rho_{0}(\phi(t)-x)-(t-\bar{t})(\dot{\phi}(t)+\mu(x))}, \quad \mu(\phi(t))=-\dot{\phi}(t),
$$

we conclude that $\theta(x) \geqslant-\mu(x)$ for $x$ close to $\phi(t)$ (see figure 4 ). It is readily verified that the solution of (3.16) is implicitly given as the solution of the algebraic equation

$$
(x-A) \mu(x)^{-a}+(t-\bar{t}) \mu(x)^{1-a}=C,
$$

where

$$
\begin{aligned}
A & =\phi(t)-\frac{(t-\bar{t})}{\rho_{0}} \dot{\phi}(t), \\
a & =\frac{\rho_{0}}{\rho_{0}-1}, \\
C & =\frac{(t-\bar{t})}{a}(-\dot{\phi}(t))^{1-a} .
\end{aligned}
$$

Let $\tilde{\mu}(z)=\mu(z+\phi(t))$ for small $z<0$ and consider the inverse function $\nu=\tilde{\mu}^{-1}$. From (3.17), we see that $\nu$ satisfies the equation

$$
\nu(y)=-\frac{(t-\bar{t})}{\rho_{0}} \dot{\phi}(t)-(t-\bar{t}) y+C y^{a}, \quad y \geqslant-\dot{\phi}(t) .
$$

A straightforward computation shows that

$$
\nu(-\dot{\phi}(t))=0, \quad \nu^{\prime}(-\dot{\phi}(t))=0, \quad \nu^{\prime \prime}(-\dot{\phi}(t))=\left(\frac{1}{1-\rho_{0}}\right) \frac{t-\bar{t}}{\dot{\phi}(t)}<0 .
$$

It follows that, for any $\alpha<0$ with $|\alpha|<\frac{1}{2}\left|\nu^{\prime \prime}(-\phi(t))\right|$, there is an $\varepsilon>0$ such that the characteristic speed $f^{\prime}(u(\cdot, t))$ satisfies the estimate

$$
0>f^{\prime}(u(x, t)) \geqslant f^{\prime}(u(\phi(t), t))-\sqrt{\frac{\phi(t)-x}{|\alpha|}} \text { for } x \in(\phi(t)-\varepsilon, \phi(t)) .
$$

In particular, choosing $\alpha=(t-\bar{t}) /\left[4\left(1-\rho_{0}\right) \dot{\phi}(t)\right]$, we obtain

$$
\sqrt{\frac{4\left(1-\rho_{0}\right)|\dot{\phi}(t)|}{t-\bar{t}}} \sqrt{\phi(t)-x} \geqslant f^{\prime}(u(\phi(t), t))-f^{\prime}(u(x, t))
$$


for all $x<\phi(t)$ sufficiently close to $\phi(t)$. We summarize the results in the following theorem.

TheOREM 3.2. Let the assumptions of theorem 3.1 hold and suppose, in addition, that the function $\rho$ defined in (3.9) satisfies property (3.10). Then, at any point $(\phi(t), t)$ on a left contact discontinuity which is not the point of intersection with another left contact discontinuity, the Hölder estimate (3.18) holds. The time $\bar{t}$ that appears in (3.18) is the time at which the maximal backward characteristic through $(\phi(t), t)$ was emitted from a contact discontinuity or, if no such exists, is zero.

We see that the coefficient in the Hölder estimate is bounded by $C / \sqrt{t-\bar{t}}$. This should be compared to the Lipschitz constant $C / t$ in the convex case (1.3), where the time $\bar{t}$ does not appear, since all characteristics can be traced back to time zero in this case. Note, however, that, also in the non-convex case, there are situations where $\bar{t}=0$, e.g. when $\phi=\phi_{l}$ is the right-most of the curves $\phi_{i}$, or when the data cross the inflection point of $f$ only once. In these cases, we have $t^{-1 / 2}$-decay of the Hölder coefficient.

\section{A generalized Oleinik estimate}

In this section we give a different generalization of the Oleinik estimate, where we have the same term of the original estimate (1.3), plus an additional one to keep into account the effect of the contact discontinuities.

Theorem 4.1. Assume that the flux $f$ is $C^{\infty}$ smooth and satisfies conditions (1.4) and (3.10). Then, for any $t>0$ and $x, y$ with $y>x$, the solution $u$ to (1.1) satisfies

$$
f^{\prime}(u(y, t))-f^{\prime}(u(x, t)) \leqslant \frac{y-x}{t}+C\left[T V u_{0}-T V u(\cdot, t)\right],
$$

where $C$ only depends on $f$ and $\left\|u_{0}\right\|_{\infty}$.

Roughly speaking, the above result is based on the following idea. As we have seen, the standard Oleinik estimate fails in the non-convex case because of the presence of contact discontinuities. On the other hand, contact discontinuities decrease the total variation of the solution, as shown by the next lemma.

Lemma 4.2. Assume the hypotheses of the previous theorem, and suppose in addition that the solution $u$ is piecewise smooth. Let

$$
\gamma_{1}:\left[\sigma_{1}, \tau_{1}\right] \rightarrow \mathbb{R}, \ldots, \gamma_{n}:\left[\sigma_{n}, \tau_{n}\right] \rightarrow \mathbb{R}
$$

be curves of a left contact discontinuity for $u$. Then we have

$$
2 \sum_{i=1}^{n}\left(\left|u\left(\gamma_{i}\left(\sigma_{i}\right)+, \sigma_{i}\right)\right|-\left|u\left(\gamma_{i}\left(\tau_{i}\right)+, \tau_{i}\right)\right|\right) \leqslant T V u(\cdot, S)-T V u(\cdot, T),
$$

where $S=\min \sigma_{i}, T=\max \tau_{i}$.

Proof. It suffices to consider the case of a single contact discontinuity curve $\gamma:[\sigma, \tau] \rightarrow \mathbb{R}$, since the proof is extended to the general case in a straightforward 
way. Let us set $u_{ \pm}(t)=u(\gamma(t) \pm, t)$ for $t \in[\sigma, \tau]$. We shall use the properties given by proposition 2.5. We know that $u_{-}(t)$ and $u_{+}(t)$ have constant signs in $[\sigma, \tau]$, one opposite to the other. Let us suppose, for instance, that $u_{+}(t)>0$ (the other case is analogous). Then $u_{+}(t)$ is a decreasing function of $t$ in $[\sigma, \tau]$, while $u_{-}(t)$ is increasing. Recalling that characteristics are emanated from $\gamma$ on the left side and go inside $\gamma$ on the right side, we deduce that, for any $t \in(\sigma, \tau)$, the function $x \rightarrow u(x, t)$ is negative and increasing in a left neighbourhood of $\gamma(t)$, jumps from $u_{-}(t)<0$ to $u_{+}(t)>0$ across $\gamma(t)$ and it is positive and decreasing in a right neighbourhood of $\gamma(t)$. Therefore, its total variation decreases in time by two times the amount $u_{+}(t)$ decreases. This proves the lemma.

Given $t \geqslant 0$ and $a<b$, we denote by $P V(a, b ; t)$ the positive variation of $x \rightarrow f^{\prime}(u(x, t))$ over the interval $[a, b]$, defined as the supremum of

$$
\sum_{i=1}^{n}\left[f^{\prime}\left(u\left(x_{i}, t\right)\right)-f^{\prime}\left(u\left(x_{i-1}, t\right)\right)\right]_{+}
$$

over all partitions $a=x_{0}<x_{1}<x_{2}<\cdots<x_{n}=b$ of the interval $[a, b]$. In the next lemma we estimate the positive variation of $f^{\prime}(u)$ in the non-convex regions.

LEMMA 4.3. Under the hypotheses of the previous lemma, we have

$$
\sum_{i=1}^{l} P V\left(\sigma_{i}(t), \phi_{i}(t) ; t\right) \leqslant C\left[T V u_{0}-T V u(\cdot, t)\right]
$$

where $C$ only depends on $f$ and $\left\|u_{0}\right\|_{\infty}$.

Proof. For simplicity of notation, we suppose that $l=1$ and we drop the subscript $i$. We consider an arbitrary partition of our interval, which we denote by $\sigma(t)=x_{0}<x_{1}<\cdots<x_{n}=\phi(t)$. By possibly refining the partition, we can assume that all points of $[\sigma(t), \phi(t)]$ lying on grazing rays are also points of the partition.

For all $k=0, \ldots, n$, let us denote by $\zeta_{k}$ the minimal backward characteristic from $\left(x_{k}, t\right)$. By definition of $\sigma(t)$, this characteristic intersects the curve $\phi$ at some time; if it is a grazing ray, it can intersect $\phi$ more than one time. We define $t_{k}^{\prime}$ to be the smallest time such that $\zeta_{k}=\phi$ and by $t_{k}^{\prime \prime}$ the largest one. Each characteristic $\zeta_{k}$ is classical in the interval $\left[t_{k}^{\prime}, t\right]$; since classical characteristics cannot intersect each other, we have $t_{k-1}^{\prime} \leqslant t_{k-1}^{\prime \prime}<t_{k}^{\prime}$ for all $k$.

We now proceed to estimate $f^{\prime}\left(u\left(x_{k}, t\right)\right)-f^{\prime}\left(u\left(x_{k-1}, t\right)\right.$ for a given $k$. Since the characteristics $\zeta_{k-1}$ and $\zeta_{k}$ are emanated from $\phi$ at time $t_{k-1}^{\prime \prime}$ and $t_{k}^{\prime}$, respectively, $\phi$ is a left contact discontinuity at these two times. We claim that $\phi$ is a left contact in the whole interval $\left[t_{k-1}^{\prime \prime}, t_{k}^{\prime}\right]$. To see this, let us call $\tau_{0}$ the smallest time such that $\phi$ is a left contact in $\left[\tau_{0}, t_{k}^{\prime}\right]$. Then, by proposition 2.5 (iv), there is a grazing ray upon $\phi$ at time $\tau_{0}$. We argue by contradiction and suppose that either $\tau_{0}=t_{k}^{\prime}$ or that $t_{k-1}^{\prime \prime}<\tau_{0}<t_{k}^{\prime}$. If $\tau_{0}=t_{k}^{\prime}$, we find that $\zeta_{k}$ grazes upon $\phi$ at time $t_{k}^{\prime}$ and this contradicts the minimality of $t_{k}^{\prime}$. If we assume instead that $t_{k-1}^{\prime \prime}<\tau_{0}<t_{k}^{\prime}$, then there exists a grazing ray lying between $\zeta_{k-1}$ and $\zeta_{k}$. Since by [6, lemma 3.5] there are no shocks in the region between $\sigma$ and $\phi$, this grazing ray is defined up to time $t$. This contradicts the assumption that our partition contains all points on grazing rays. Thus we have proved our claim that $\phi$ is a left contact in $\left[t_{k-1}^{\prime \prime}, t_{k}^{\prime}\right]$. 
For simplicity, let us now set $\left.u_{ \pm}(t)=u(\phi(t) \pm, t)\right)$. Then, recalling that $\left|u_{+}(t)\right|$ and $\left|u_{-}(t)\right|$ are both decreasing by proposition 2.5 (ii), and using properties (3.9) and (3.10), we obtain

$$
\begin{aligned}
f^{\prime}\left(u\left(x_{k}, t\right)\right)-f^{\prime}\left(u\left(x_{k-1}, t\right)\right) & =f^{\prime}\left(u_{-}\left(t_{k}^{\prime}\right)\right)-f^{\prime}\left(u_{-}\left(t_{k-1}^{\prime \prime}\right)\right) \\
& =\rho\left(u_{-}\left(t_{k}^{\prime}\right)\right) f^{\prime}\left(u_{+}\left(t_{k}^{\prime}\right)\right)-\rho\left(u_{-}\left(t_{k-1}^{\prime \prime}\right)\right) f^{\prime}\left(u_{+}\left(t_{k-1}^{\prime \prime}\right)\right) \\
& \leqslant \rho\left(u_{-}\left(t_{k}^{\prime}\right)\right)\left[f^{\prime}\left(u_{+}\left(t_{k}^{\prime}\right)\right)-f^{\prime}\left(u_{+}\left(t_{k-1}^{\prime \prime}\right)\right)\right] \\
& \leqslant M\left[\left|u_{+}\left(t_{k-1}^{\prime \prime}\right)\right|-\left|u_{+}\left(t_{k}^{\prime}\right)\right|\right],
\end{aligned}
$$

where we have set

$$
M=\max \left\{\left|f^{\prime \prime}(v)\right|:|v| \leqslant\left\|u_{0}\right\|_{\infty}\right\} \cdot \max \left\{\rho(v):|v| \leqslant\left\|u_{0}\right\|_{\infty}\right\} .
$$

Summing over $k$, using lemma 4.2 and recalling that the partition was arbitrary, we obtain

$$
P V(\sigma(t), \phi(t) ; t) \leqslant \frac{1}{2} M\left[T V u_{0}-T V u(\cdot, t)\right]
$$

Proof of theorem 4.1. Let us first consider the case when the solution is piecewise smooth. Then we can estimate $P V(a, b ; t)$ using proposition 2.4 in the convex regions and lemma 4.3 in the non-convex ones. More precisely, we choose points $a=x_{0}<x_{1}<\cdots<x_{m}=b$ in such a way that each subinterval $\left[x_{j-1}, x_{j}\right]$ is either contained in some $\left[\phi_{i-1}(t), \sigma_{i}(t)\right]$ or in some $\left[\sigma_{i}(t), \phi_{i}(t)\right]$. In the first case, we deduce from proposition 2.4 that $P V\left(x_{j-1}, x_{j} ; t\right) \leqslant\left(x_{j}-x_{j-1}\right) / t$, while the total contribution of the intervals of the latter type is bounded by $C\left[T V u_{0}-T V u(\cdot, t)\right]$ by virtue of lemma 4.3. Summing over $j$, we obtain

$$
P V(a, b ; t) \leqslant \frac{b-a}{t}+C\left[T V u_{0}-T V u(\cdot, t)\right] .
$$

Since $f^{\prime}(u(b, t))-f^{\prime}(u(a, t)) \leqslant P V(a, b ; t)$, this proves the assertion in the case when $u$ is piecewise smooth. The general case can be recovered by a standard approximation procedure, using the property that the solutions to (1.1) are generically piecewise smooth by theorem 2.1 .

COROLlary 4.4. Let the assumptions of the previous theorem be satisfied. Then, for every $\varepsilon>0$, the positive variation over $\mathbb{R}$ of the function

$$
x \rightarrow f^{\prime}(u(x, t))-(1+\varepsilon) \frac{x}{t}
$$

tends to zero as $t \rightarrow+\infty$.

Proof. Let us take $t_{0}, t$ with $t>t_{0}>0$. By taking $u\left(\cdot, t_{0}\right)$ as initial data, we can generalize (4.3) as follows:

$$
P V(a, b ; t) \leqslant \frac{b-a}{t-t_{0}}+M\left[T V u\left(\cdot, t_{0}\right)-T V u(\cdot, t)\right]
$$

Since $T V u(\cdot, t)$ is positive and decreasing in $t$, the term $T V u\left(\cdot, t_{0}\right)-T V u(\cdot, t)$ can be made arbitrarily small by taking $t_{0}$ sufficiently large. Also, for fixed $t_{0}$, we have $(b-a) /\left(t-t_{0}\right)<(1+\varepsilon)(b-a) / t$ if $t$ is large enough. The assertion follows. 
Let us remark that the standard Oleinik estimate (1.3) is equivalent to the property that the function

$$
x \rightarrow f^{\prime}(u(x, t))-\frac{x}{t}
$$

has zero positive variation. Therefore, the above corollary says, roughly speaking, that the Oleinik inequality is asymptotically valid also in the non-convex case considered in this paper.

\section{Acknowledgments}

The authors are indebted to N. H. Risebro, C. Tegnander and F. Bouchut for discussions.

\section{References}

1 A. Bressan and R. Colombo. Decay of positive waves in nonlinear systems of conservation laws. Ann. Scuola Norm. Sup. Pisa 26 (1998), 133-160.

2 A. Bressan and P. G. LeFloch. Structural stability and regularity of entropy solutions to hyperbolic systems of conservation laws. Indiana Univ. Math. J. 48 (1999), 43-84.

3 K.-S. Cheng. A regularity theorem for a non-convex scalar conservation law. J. Diff. Eqns 61 (1986), 79-127.

4 C. Cheverry. Regularizing effects for multidimensional scalar conservation laws. Ann. Inst. Henri Poincaré 17 (2000), 413-472.

5 C. M. Dafermos. Characteristics in hyperbolic conservation laws. A study of the structure and the asymptotic behavior of solutions. In Nomlinear analysis and mechanics: HeriotWatt Symposium (ed. R. J. Knops). Pitman Research Notes in Mathematics, vol. 1 (London: Pitman, 1977).

6 C. M. Dafermos. Regularity and large time behavior of solutions of a conservation law without convexity. Proc. $R$. Soc. Edinb. A 99 (1985), 201-239.

7 A. F. Filippov. Differential equations with discontinuous righthand sides (Dordrecht: Kluwer Academic, 1988).

8 J. Glimm and P. D. Lax. Decay of solutions of systems of nonlinear hyperbolic conservation laws. Mem Am Math. Soc. 101 (1970).

9 D. Hoff. The sharp form of Oleinik's entropy condition in several space variables. Trans. Am Math. Soc. 276 (1983), 707-714.

10 E. Hopf. The partial differential equation $u_{t}+u u_{x}=\mu u_{x x}$. Commom. Pure Appl. Math. 3 (1950), 201-230.

11 S. N. Kružkov. First order quasilinear equations in several independent variables. Mat. Sb. 81 (1970), 228-255. (Transl. Mat. USSR Sb. 10 (1970), 217-243.)

12 O. Oleinik. Discontinuous solutions of nonlinear differential equations. Usp. Mat. Nauk 12 (1957), 3-73. (Transl. Am Math. Soc. Transl. Ser. 2 26, 95-172.)

13 C. Sinestrari. Instability of discontinuous traveling waves for hyperbolic balance laws. $J$. Diff. Eqns 134 (1997), 269-285.

14 E. Tadmor. Local error estimates for discontinuous solutions of nonlinear hyperbolic equations, SIAM J. Numer. Analysis 28 (1991), 891-906.

15 C. Tegnander. Conservation laws with non-convex flux and application to two-phase flow in porous media. PhD thesis, University of Oslo (1998).

16 K. Zumbrun. Decay rates for non-convex systems of conservation laws. Commun. Pure Appl. Math. 46 (1993), 353-386.

\section{(Issued 17 August 2001)}

